\title{
Effects of Aerobic Training on the Microcirculation: A Mini Review
}

\author{
Saghiv $\mathbf{M}^{1 *}$, Welch $\mathrm{L}^{2}$ and Sawhney $\mathbf{M}^{3}$ \\ ${ }^{1}$ Department of Human Performance and Leisure Studies, USA \\ ${ }^{2}$ Department of Kinesiology, USA \\ ${ }^{3}$ Department of Psychology, USA
}

*Corresponding author: Moran Sciamama Saghiv, Associate Professor of Clinical Exercise Physiology Chair, Department of Human Performance and Leisure Studies, College of Health and Human Sciences, NC A \&T State University, USA.

Received Date: December 19, 2018

Published Date: January 09, 2019

\begin{abstract}
The microcirculation is composed of four different sections: arterioles, capillaries, sphincters and venules. The coronary arteries microcirculation consists the heart and large coronary arteries. The myocardium almost solely dependent on the ability of aerobic metabolism, therefore, oxygen demand must be balance by increasing coronary blood flow compared to rest, almost six-folds at maximal oxygen uptake. Increase in skeletal muscle oxygen demand is met by increasing oxygen delivery namely cardiac output, muscle's oxygen extraction and microcirculation blood flow. However, at rest oxygen extraction by the myocardium is maximal, hence the only way to balance oxygen demand during exercise is by linear increase in coronary blood flow. Repeated aerobic exercise changes structural and functional adaptations within the cardiovascular system include increases myocardial capillary density, increase vascularity diameters and changes in cell shape and cytoskeletal rearrangement. It is our aim to discuss these numerous contributions on coronary blood flow. We also have extended our discussion to the effect of chronic aerobic exercise which modify some controlling developments in the coronary microcirculation Red blood cells.
\end{abstract}

Keywords: Microcirculation; Dynamic exercise; Arterioles; Myocardium; Red blood cell deformability

\section{The Microcirculation Webs}

Microcirculation means blood flow through the smallest vessels, within organ tissues and is the link between blood and single cell. In addition, the microcirculation delivers oxygen and nutrients and remove

carbon dioxide. The microcirculation is the largest components in the cardiovascular system, and is composed of four different sections: arterioles, capillaries, sphincters and venules [1]. Each component has sole assembly and purpose. The flow of blood through the microvasculature, is in vessels with diameter less then $120 \mu \mathrm{m}$, within organ tissues. It is composed of terminal arterioles contracted by sympathetic nerves with a diameter of $75 \mu \mathrm{m}$ [2]. The arteriole regulates blood flow and thus, influencing blood pressure and consequently, total peripheral resistance [3]. The sphincters before the capillaries still with small amount of smooth muscle respond to chemical compounds as the autoregulation mechanism [4]. Blood flow continues through the capillary bed with diameter of $7 \mu \mathrm{m}$ almost as the red blood cell size, and intima tone with thin wall that facilitates exchange of gasses by diffusion. Then, blood flows to the venules with a diameter of $120 \mu \mathrm{m}$. The major site of exchange of materials occur in the capillaries and in the junction of capillary-venules [5].

\section{The Coronary Arteries Microcirculation}

It consists the heart and large coronary arteries. When the arteries are occluded, myocardial infarction might happen. The coronary microcirculation has an important role to maintain oxygen demand-supply balance. To achieve this, it will be essential that the myocardium's coronaries resistance will integrate the involvement of inherent and extrinsic signals. The myocardium almost solely dependent on the ability of aerobic metabolism [6], with maximal oxygen extraction at rest. On the other hand, the anaerobic pathway is inadequate to produce energy. With increase left ventricular contractility and heart beats demand for ATP and therefore, oxygen is matched by myocardial aerobic metabolism due, to large and exceptional amounts of myocardial mitochondria. 
Since at rest oxygen extraction by the myocardium is maximal, the only way at maximal oxygen uptake to balance the increased myocardial oxygen demand is by linear increase in coronary blood flow almost six-folds [7].

The oxidative production of ATP is mainly achieved from fatty acids in the Tricarboxylic acid cycle [6]. However, in normal subjects, increase in myocardial oxygen demand to maintain viability, is usually, balanced and met by augmentation oxygen delivery. Coronary blood flow is normally regulated by resistance in the arterioles of the coronary bed. Flow resistance in the coronary circulation has mixture signals coming from small arteries and arterioles. Resistance in the arterioles of the coronary are influenced mainly by: myogenic activity, metabolic activity influencing the autoregulation mechanism, neurological and humoral factors, and by the endothelium. The greatest important and leading mechanism is the metabolic one. The oxygen requirements of the myocardium control the coronaries diameter thus, coronary artery diameter is increased by the vasodilation mechanism [7]. Normal arteries are capable to maintain the coronary blood flow at levels appropriate to the needs of the myocardium by the autoregulation mechanism. Myogenic and endothelial regulatory mechanisms effects on coronary diameters by stretch. Myogenic coronary arterioles narrow and then dilate while shear stress induced dilation [8]. The vascular myogenic response refers to the acute reaction of a blood vessel to a change in transmural pressure. This response is critically important for the development of resting vascular tone [8].

So, in healthy individuals, coronary blood flow does not limit aerobic metabolism of the myocardium during maximal aerobic exercise [9].

\section{Red Blood Cell Mechanical Properties}

Red blood cell is involved through number mechanisms in order to increase oxygen delivery as needed by the working muscles during aerobic exercise. The increase in skeletal muscle demand is met by increasing oxygen delivery namely cardiac output, increases in muscle's oxygen extraction and increasing microcirculation blood flow [10]. Associated with this there is an increased release into the circulation of catecholamine. The large fraction of the blood volume is contained in the microcirculation webs and are affected as well. These alterations of blood rheology are related to muscular activity and intensity [11]. These may give rise in the traffic of Thallium 201while testing vascularity changes for myocardial perfusion.

The mechanical properties and filterability of circulating red blood cells play a central role in the function of the microcirculation [12]. Cell membrane mechanical fluctuation of red blood cells plays a vital role in health and disease. Circulatory resistance depends mainly on red cell concentration, plasma viscosity and on red cell deformability [12]. The ability of red blood cells to deform is crucial to allow their circulation, especially when passing in narrow capillaries this in turn, determine vessel's resistance to flow [13].

Catecholamine appears to play an important role in membrane fluctuations and filterability of red blood cells due to changes in lymphocyte levels which are dependent on $\beta$-adrenergic receptor expression on the respective cell types and also on cyclic adenosine monophosphate (cAMP) which activates protein kines A. It was shown that following the administration of prostaglandin E1 and adrenaline cell membrane fluctuations and filterability of red blood cells were elevated [14], through a cAMP-dependent pathway.

\section{Effect of Aerobic Exercise Training}

It is type of dynamic exercise low to high intensity that relies largely on the Krebs aerobic pathway to produce ATP [15]. Thus, forming ATP is mainly done with the present of oxygen [16]. Aerobic exercise is characterized by sustained and prolonged activity that can last from few minutes up to hours [15], whereas vasculature play a critical role in the physiological adaptations of the myocardium in response to physical exercise. The practical measurements of the cardiopulmonary system is the aerobic capacity measured as maximal oxygen uptake.

Based on evidence that chronic repeated aerobic exercise changes structural and functional adaptations within the cardiovascular system [17] it: a. increases myocardial capillary density; b. increase vascularity diameters [18] and therefore, reduces resistance of coronary blood flow that is greatest in the coronary arterioles smooth muscle c. change red blood cell mechanical properties [19] namely, change in cell shape and cytoskeletal rearrangement. Following aerobic exercise training adaptations in the coronary microcirculation increase the capacity of the coronaries blood flow [20]. Therefore, the microcirculation network stimulates erythropoiesis and consequently, augments blood hemoglobin levels and red blood cells [21]. Aerobic exercise and training regimens have large and lasting effects on hemoglobin, by altering plasma levels and augment its levels [22,23]. This, in turn increases the ability of the blood to carry larger amount of oxygen to the working muscles and myocardium [24].

In addition, benefits of aerobic exercise include; increase in myocardial contractility ability and cardiovascular system, improve oxygen delivery-extraction, rise in energy availability, increase endurance with low lactic acid levels, reduce cardiac risk factors and growth in lean body mass, increase blood supply and therefore oxygen and nutrients to better functioning cell and, then the organ is more efficient. Acute exercise disrupts hemorheological in a high exercise intensity however, more important is hemoconcentration. The rheological properties of blood may control, in part, the alteration rate of oxygen delivery-supply at the onset of exercise [25].

\section{Conclusion}

Following long-lasting high aerobic exercise intensity training, in well-trained subjects erythrocyte deformability were unchanged following test to maximal oxygen uptake [26]. It has been suggested that the reduced oxygen content in the arterials due to strenuous exercise [27], in well-trained subjects is a positive adaptive mechanism that facilitate the muscle's contractions. However, significant increase in erythrocyte deformability response was found in well-trained athletes following submaximal running of four periods of sub-maximal running at $70 \%$ of peak oxygen uptake for $30 \mathrm{~min}$ [28]. Therefore, it can be suggested that the effect of submaximal aerobic exercise bouts at $70 \%$ of maximal oxygen 
uptake influence red blood cell deformability [29]. On the other hand, red blood cell deformability does occur under strenuous aerobic exercise in well trained athletes.

\section{Acknowledgement}

None.

\section{Conflict of Interest}

No conflict of interest.

\section{References}

1. Li L, Gao XL, Song YZ, Xu H, Yu GY, et al. (2007) Anatomy of Arteries and Veins of Submandibular Glands. Chin Med J (Engl) 120: 1179-1182.

2. Kuo L, Davis MJ, Chilian WM (1988) Myogenic Activity in Isolated Subepicardial and Subendocardial Coronary Arterioles. Am J Physiol 255: H1558-H1562.

3. Hong K, Li M, Nourian Z, Meininger GA, Hill MA, et al. (2017) Angiotensin II Type 1 Receptor Mechanoactivation Involves RGS5 (Regulator of G Protein Signaling 5) in Skeletal Muscle Arteries: Impaired Trafficking of RGS5 in Hypertension. Hypertension 70: 1264-1272.

4. Ping P, Johnson PC (1992) Role of Myogenic Response in Enhancing Autoregulation of Flow during Sympathetic Nerve Stimulation. Am J Physiol 263: H1177-1184.

5. Piagnerelli M, Ince C, Arnaldo Dubin A (2012) Microcirculation. Crit Care Res Pract 2012: 867176.

6. Weiss RG, Maslov M (2004) Normal Myocardial Metabolism: Fueling Cardiac Contraction. Adv Stud Med 4: S457-S463.

7. Duncker DJ, Bache RJ (2008) Regulation of Coronary Blood Flow during Exercise. Physiol Rev 88: 1009-1086.

8. Davis MJ, Hill MA (1999) Signaling Mechanisms Underlying the Vascular Myogenic Response. Physiol Rev 79: 387-423.

9. Laughlin MH (2004) Physical Activity in Prevention and Treatment of Coronary Disease: The Battle Line is in Exercise Vascular Cell Biology. Med Sci Sports Exerc 36: 352-362.

10. Laughlin MH, Davis MJ, Secher NH, van Lieshout JJ, Arce-Esquivel AA, et al. (2012) Peripheral Circulation. Compr Physiol 2: 321-447.

11. Brun JF, Varlet-Marie E, Connes P, Aloulou I (2010) Hemorheological alterations related to training and overtraining. Biorheology 47(2): 95-115.

12. Stuart J, Nash GB (1990) Red Cell Deformability and Haematological Disorders. Blood Rev 4: 141-147.

13. Nash GB (1991) Red Cell Mechanics: What Changes are needed to Adversely Affect in Vivo Circulation. Biorheology 28: 231-239.
14. Muravyov AV, Tikhomirova I (2013) Role Molecular Signaling Pathways in Changes of Red Blood Cell Deformability. Clin Hemorheol Microcirc 53: 45-59.

15. Plowman SA, Smith DL (2007) Exercise Physiology for Health, Fitness, and Performance. Daryl Fox: 1750.

16. McArdle WD, Katch FI, Katch VL (2006) Essentials of Exercise Physiology. Lippincott Williams \& Wilkins P: 204

17. Huonker M, Halle M, Keul J (1996) Structural and Functional Adaptations of the Cardiovascular System by Training. Int J Sports Med 3: S164-S172.

18. Leung FP, Yung LM, Laher I, Yao X, Chen ZY, et al. (2008) Exercise, Vascular Wall and Cardiovascular Diseases. Sports Medicine 38: 10091024.

19. Yalcin O, Bor-Kucukatay M, Senturk UK, Baskurt OK (2000) Effects of Swimming Exercise on Red Blood Cell Rheology in Trained and Untrained Rats. J Appl Physiol 88: 2074-2080.

20. Laughlin MH, Bowles DK, Duncker DJ (2012) The Coronary Circulation in Exercise Training. J Physiol Heart Circ Physiol 302: H10-23.

21. Hu M, Lin W (2012) Effects of Exercise Training on Red Blood Cell Production: Implications for Anemia. Acta Haematol 127: 156-164.

22. Brun JF, Varlet-Marie E, Connes P, Aloulou I (2010) Hemorheological Alterations Related to Training and Overtraining. Biorheology 47: 95115.

23. Mairbauri H (2013) Red Blood Cells in Sports: Effects of Exercise and Training on Oxygen Supply by Red Blood Cells. Frontiers in Physiology 4:332.

24. Montero D, Cathomen A, Jacobs RA, Flück D, de Leur J, et al. (2015) Haematological Rather than Skeletal Muscle Adaptations Contribute to the Increase in Peak Oxygen Uptake Induced by Moderate Endurance Training. J Physiol 593: 4677-4688.

25. Simmonds MJ, Tripette J, Sabapathy S, Marshall Gradisnik SM, Connes P, et al. (2011) Cardiovascular Dynamics During Exercise are Related to Blood Rheology. Clin Hemorheol Microcirc 49: 231-241.

26. Alis R, Sanchis-Gomar F, Ferioli D, La Torre A, Blesa JR, et al. (2015) Exercise Effects on Erythrocyte Deformability in Exercise-Induced Arterial Hypoxemia. Int J Sports Med 36: 286-291.

27. Harms CA, McClaran SR, Nickele GA, Pegelow DF, Nelson WB, et al. (1998) Exercise-Induced Arterial Hypoxaemia in Healthy Young Women. J Physiol 507: 619-628.

28. Wahl P, Bloch W, Mester J, Born DP, Sperlich B, et al. (2012) Effects of Different Levels of Compression during Sub-Maximal and HighIntensity Exercise on Erythrocyte Deformability. Eur J App l Physiol 112: 2163-2169.

29. Simmonds MJ, Connes P, Sabapathy S (2013) Exercise-Induced Blood Lactate Increase does not Change Red Blood Cell Deformability in Cyclists. PLoS One 8(8): e71219. 